\title{
Restructurations d'espaces militaires et développement des territoires
}

Military restructuring and territorial development

Militärische Umstrukturierung und territoriale Entwicklung

\section{Pierre Ginet}

\section{OpenEdition}

\section{Journals}

Édition électronique

URL : http://journals.openedition.org/rge/3219

DOI : $10.4000 /$ rge.3219

ISSN : 2108-6478

\section{Éditeur}

Association des géographes de l'Est

Édition imprimée

Date de publication : 30 mai 2011

ISSN : 0035-3213

\section{Référence électronique}

Pierre Ginet, «Restructurations d'espaces militaires et développement des territoires », Revue Géographique de l'Est [En ligne], vol. 51 / 1-2 | 2011, mis en ligne le 18 janvier 2012, consulté le 08 septembre 2020. URL : http://journals.openedition.org/rge/3219; DOI : https://doi.org/10.4000/rge. 3219

Ce document a été généré automatiquement le 8 septembre 2020.

Tous droits réservés 


\title{
Restructurations d'espaces militaires et développement des territoires
}

\author{
Military restructuring and territorial development \\ Militärische Umstrukturierung und territoriale Entwicklung
}

\author{
Pierre Ginet
}

1 Ce numéro de la Revue Géographique de l'Est prend sa source dans les réflexions menées à Metz par l'équipe de recherche GEOPOL sur les questions reliant défense et aménagement. Un champ de recherche exploré dès les années 1980 et débouchant par ailleurs sur un cursus de formation de troisième cycle qui correspond à l'actuelle spécialité de Master DEMETERR «Défense - Méthodes - Territoires». Cet intérêt scientifique et pédagogique exprimé par plusieurs générations de géographes messins n'est pas dû au hasard. La Lorraine forme un terrain d'études exceptionnel pour l'approche de ces questions. Héritière de l'ancienne Lotharingie, elle constitue une zone-frontière façonnée par la collision récurrente de forces armées venues d'est et d'ouest s'affronter ici depuis plus de mille ans. A la marge de territoires aux identités très affirmées, elle est devenue lieu d'enjeu majeur en termes de conquête ou de défense territoriale. Sa situation frontalière a fait cristalliser ici plus qu'ailleurs le sentiment de l'identité des peuples et la détermination inflexible et cruelle conduisant à son affirmation conquérante ou à sa défense légitime. Plus que dans d'autres régions, l'identité lorraine s'est forgée par le retour régulier de la guerre et par l'omniprésence $\mathrm{du}$ fait militaire. Partout des casernes, des hommes en uniforme, des forts, des régiments de blindés à l'entraînement, des avions de chasse patrouillant à basse altitude... La guerre y a trop longtemps été préparée, y est trop souvent revenue. Absente depuis trois générations, son souvenir, ses séquelles et ses artefacts demeurent pourtant bien ancrés dans les mentalités et les paysages.

2 L'année 2008 marque cependant un tournant dans cette histoire longue. Le dernier Livre blanc sur la défense et la sécurité nationale amorce en effet le reflux de la présence militaire. Les suppressions d'emplois militaires et civils du Ministère de la 
défense et la cession des terrains appartenant à ce dernier doivent permettre d'adapter l'organisation des forces à l'évolution de la menace comme aux contraintes budgétaires de l'Etat et à la perspective d'un transfert progressif de la charge de la défense nationale à l'échelon européen et à l'OTAN. Un ajustement drastique qui aura un impact très lourd sur l'économie, la démographie et l'identité de la Lorraine, région française la plus touchée par la réforme des Armées.

3 A quelques années du centenaire de la Grande guerre et de la bataille de Verdun, alors que la mémoire s'étiole, les acteurs du monde politique lorrain s'interrogent sur les moyens qui permettraient de valoriser un patrimoine dont la portée symbolique est universelle. Ils doivent dans le même temps faire face à la prise en charge d'emprises foncières et immobilières trop nombreuses pour être rapidement intégrées aux processus de recomposition territoriale auxquels participent les aménageurs dans leur souci de développer les portions d'espace dont ils ont la responsabilité.

Dans le quart nord-est de la France, une trentaine de sites militaires sont ainsi directement concernés par la dernière réforme des Armées. Leurs personnels doivent être redéployés et leurs infrastructures reprises par différents ministères ou transmises aux agglomérations les plus pénalisées par ces fermetures. Loin d'apparaître comme une manne foncière providentielle lorsque ces terrains sont cédés pour l'euro symbolique, leur vente aux collectivités locales ou aux investisseurs privés s'avère difficile, faute de moyens financiers disponibles et de projets territoriaux suffisamment consensuels, ambitieux ou rentables. J-L.CHINOUILH, général de corps d'armée, gouverneur militaire de Metz, commandant la Région Terre Nord-Est et officier général de la zone de défense Est a pris en charge la lourde responsabilité de ce dossier jusqu'en 2010. Il nous explique que le processus de cession se déroule de façon satisfaisante, mais que les possibilités de reclassement des emprises se réduisent au fil du temps.

Que faire alors de cet héritage singulièrement encombrant? Une fois en leur possession, c'est la capacité tant visionnaire que financière des collectivités territoriales qui est interpellée. Les détruire totalement ou partiellement ? En valoriser la portée mémorielle? Les conserver en attendant une solution? Les dépecer morceau par morceau au gré de projets décousus ou planifier un grand projet associant de nombreux acteurs dans une logique de développement relevant de la métropolisation ?... Les potentialités sont nombreuses tout comme le sont les questions préalables auxquelles il faudra bien répondre: Quels acteurs mobiliser? Qui va payer? A quelle hauteur, sachant que les postes sont nombreux (gardiennage, dépollution, destruction...)? Quel mode de gouvernance de projet mettre en place? Comment articuler les échelles ? Et finalement, les acteurs lorrains sont-ils capables de se saisir et de traiter collectivement cette question? A moins que cette dernière soit le dernier révélateur de leurs insolubles dissensions... Car le devenir de ces espaces militarisés résulte d'abord de choix politiques locaux et d'une capacité à associer les points de vue souvent contradictoires des acteurs publics lorrains et de l'Etat. Derrière la fermeture des casernes partiellement compensée par des mesures exceptionnelles fournies par ce dernier, une instrumentalisation des décideurs lorrains doit dès lors ne pas être écartée. L'analyse géopolitique que propose P.GINET, professeur des universités à Metz, tente de soulever les termes et de vérifier la pertinence d'une telle hypothèse.

6 Force est de constater comme le fait D.MATHIS, docteur en géographie à Nancy, que le redéploiement des régiments des villes de garnison est à l'origine d'un profond malaise et de fortes oppositions. L'analyse géohistorique qu'il consacre au sud mosellan 
démontre que les différentes vagues du déploiement militaire dans cet espace ont servi de ferment à l'émergence puis à la consolidation d'identités locales fortes, formalisées dans l'objet géographique qu'est la ville de garnison. Elle démontre également que l'identité historique persiste bien après que la fonction militaire qui en est à l'origine ait disparu. Cette approche géohistorique permet de comprendre les spécificités des villes de garnison. Elle souligne les formes multiples de restructurations qui découlent de la restructuration, sans préjuger de la pertinence que ce souhait de ne pas rompre avec le passé présente, au regard de la nécessité d'un nouveau développement des territoires.

7 Mais la restructuration militaire prévue par la réforme de 2008 concerne la totalité du territoire national et ne se réduit pas à la fermeture de sites militaires. Elle se traduit aussi par l'aménagement de nouveaux sites. Cette production territoriale, toutefois de moindre ampleur, se concrétise par la mise en place des bases de défense, analysée par F.RAPIN, doctorant en géographie au laboratoire Espaces \& Sociétés de l'université d'Angers à partir de la situation de la façade ouest de la France.

8 La question des restructurations d'espaces militaires place le géographe face à des enjeux dont la portée dépasse le cas du seul territoire français. Le renouvellement territorial d'espaces militaires constitue un sujet d'actualité et pour tout dire intemporel, à l'échelle mondiale, que ce dernier suive immédiatement le conflit ou intervienne plus tardivement. L'homo bellicus à l'œuvre laisse de tout temps et en tous lieux une empreinte géographique spécifique dont il s'agit, une fois la paix rétablie, de définir la réaffectation fonctionnelle, les modalités de recyclage territorial, le réaménagement.

9 Même lorsqu'ils sont anciens, les stigmates de la guerre et de la militarisation de l'espace demeurent encore douloureux. L'heure n'est plus alors au diagnostic et à l'élaboration de scénarios de redéveloppement des territoires, mais à la mise en œuvre de projets chargés de concrétiser ces visions stratégiques. C'est la réflexion que nous propose A.N'DAYE, lieutenant-colonel de la Force de Défense Nationale du Burundi et conseiller principal auprès du ministère burundais de la défense nationale et des anciens combattants, au travers de l'analyse des restructurations militaires induites par la formation de la Force de Défense Nationale burundaise qui fait suite à la militarisation de l'espace des grands lacs dans le cadre du conflit Hutu-Tutsi.

10 Le recyclage des espaces militaires pose des questions non seulement stratégiques et politiques mais aussi techniques et économiques. La géographie, au service du déroulement de la guerre met ensuite ses outils à la disposition des aménageurs pour en traiter les traces et favoriser un nouveau développement, forcément durable. Le processus de renouvellement territorial observable dans le Chatt-el-Arab, durement touché par les guerres Iran-Irak illustre cette position ambivalente d'une géographie technicienne et sans états d'âme, tantôt au service de l'art de la guerre, tantôt œuvrant à la paix, mais toujours complaisante et précieuse conseillère du prince. A.LIEGE, professeur de lycée à Toulouse et auteur d'une thèse sur cette région nous propose son analyse en mettant l'accent sur la manière dont les techniques de l'information utilisées pour la gestion d'un champ de bataille, servent de nos jours à aider la reconstruction d'un espace à la fois écosystème fragile et zone économique vitale pour l'équilibre géopolitique de la région.

11 Enfin, avec plus de 600 fortifications, la région frontalière Saar-Lor-Lux offre une grande variété de formes d'architecture militaire. Aucune autre région n'offre un panel 
aussi représentatif de l'ensemble des étapes de l'histoire de la castramétration au regard de l'amélioration concomitante de la poliorcétique. Pourtant, la perception sociétale de ces fortifications varie fortement. En France et au Luxembourg, ces dernières sont considérées comme un héritage de grande valeur et plusieurs d'entre elles sont d'ailleurs classées au titre du patrimoine mondial de l'UNESCO. Leur reconnaissance comme éléments de l'héritage commun ne prête pas à polémique et plusieurs d'entre elles sont valorisées en tant que telles. Mais en Allemagne, la situation est totalement opposée. La perception positive des fortifications dans l'opinion publique demeure une exception. I.EBERLE et A.REICHERT-SCHIK, professeur des universités et enseignant-chercheur à l'Université de Trêves, analysent le traitement des fortifications dans différents secteurs de la région frontalière Allemagne-FranceLuxembourg et tentent d'expliquer quels partis d'aménagement peuvent servir à renforcer l'acceptabilité sociale des fortifications militaires en tant qu'héritage culturel, en particulier au travers d'actions ciblées sur un développement et un aménagement touristique à la fois durable et transfrontalier.

Les nombreux et difficiles défis soulevés par la reconversion d'espaces militaires relève d'une alchimie qui mêle étroitement technicité, analyse géopolitique, capacité à articuler les échelons territoriaux, sens de l'histoire et vision prospective. Le géographe occupe une place privilégiée dans la pratique de cet art de l'aménagement auxquels les décideurs politiques devront encore faire appel pour produire cette transmutation seule à même d'engager leurs territoires dans la spirale vertueuse du développement.

\author{
AUTEUR \\ PIERRE GINET \\ CEGUM - Université Paul Verlaine - Ile du Saulcy, 57045 Metz CEDEX
}

